\title{
High Temperature Stability and Performance Analysis of N-doped Ge-Se-Sb based OTS Selector Devices
}

\author{
A. Verdy, G. Navarro, M. Bernard, P. Noé, C. Licitra, \\ G. Bourgeois, J. Garrione, M.C. Cyrille, V. Sousa, E. Nowak \\ CEA, LETI, MINATEC Campus, 17 rue des Martyrs, 38054 Grenoble Cedex 9, France
}

\begin{abstract}
In this paper, we investigate the stability at high temperature of $\mathrm{N}$-doped Ge-Se-Sb based Ovonic Threshold Switching selectors (OTS). Annealing temperatures up to $400^{\circ} \mathrm{C}$ are explored, compatible with IC Back-End-Of-Line temperature budget (BEOL). Thanks to electrical characterization and material physico-chemical analysis, we show how an annealing of 30 minutes at $400^{\circ} \mathrm{C}$ is beneficial for the reduction of the threshold voltage. Moreover, after such thermal budget, an ultra-low leakage current of $0.1 \mathrm{nA}$ at $\mathrm{V}_{\mathrm{th}} / \mathbf{2}$ and an endurance of more than $10^{8}$ cycles are still ensured. Electrical parameters and selector performance are then analyzed at stress temperatures up to $150^{\circ} \mathrm{C}$, demonstrating the suitability of Ge-Se-Sb based OTS selector devices for BEOL integrations for applications targeting high temperature operating environments.
\end{abstract}

Index Terms-OTS, BEOL selector, thermal stability, N-doped Ge-Se-Sb, high temperature.

\section{INTRODUCTION}

Resistive Crossbar arrays are attracting great interest to achieve memory architectures featuring high density and high endurance performance, in particular to address Storage Class Memory applications (SCM). In a Crossbar array, the memory cells are connected together in parallel along wordlines and bitlines. In order to hinder sneak-paths and cell-to-cell cross programming/reading disturbs, that not even optimized polarization techniques can completely overcome [1], a selector device (1S) added in series with the memory device (1S1R) becomes mandatory. Strong non-linearity, high endurance, low leakage current and Back-End-Of-Line (BEOL) thermal budget compatibility, are some of the main challenges faced by such device. Among the different selector technologies [2], the one based on the Ovonic Threshold Switching (OTS) [3] is one of the most promising, confirmed by the recent reverse-engineering of the Intel's Optane product [4].

OTS devices are based on highly resistive amorphous chalcogenide materials, in which their selectivity rely on the electrical transition between a low-conductive state (OFF-state) and a metastable conductive state (ON-state). This transition (switch) is achieved when the voltage crosses the Threshold Voltage $\left(\mathrm{V}_{\text {th }}\right)$. However, the high $\mathrm{ON}$-current density during programming operations (inducing Joule heating effect) and the BEOL thermal budget (up to temperatures higher than $400^{\circ} \mathrm{C}$ ) can lead to segregation phenomena and/or the crystallization of the amorphous material, affecting its functionality. Recent studies on Tellurium-based OTS devices revealed the difficulty to find an optimum solution combining at the same time thermal stability and threshold switching reliability [5]. On the contrary,
Selenium-based chalcogenides represent a valid class of materials to target selector applications, thanks to their higher glass forming ability wrt Te-based alloys [6]. In particular, Ge-Se based OTS devices showed a high thermal stability that can be even improved thanks to the incorporation of dopants, responsible for the creation of strong covalent bonds, as demonstrated with $\mathrm{N}$ doping [7]. Nevertheless, a detailed study on the impact of the thermal budget and on high temperature operations on the electrical performance of such OTS materials has not yet been reported in the literature. Therefore, we present in this paper the thermal stability analysis of N-doped Ge-Se-Sb based OTS selectors. We report the electrical parameters evolution at different annealing temperatures (up to $400^{\circ} \mathrm{C}$ ) and durations ( $>1$ hour), and with the support of physico-chemical characterizations (spectroscopic ellipsometry, X-Ray diffraction, Infrared and Raman spectroscopy), we highlight the main structural changes that take place in the material. Finally, annealed OTS devices performance is investigated through the analysis of the threshold voltage, of the conduction mechanism, of the endurance and of the leakage current at room temperature and under temperature stress up to $150^{\circ} \mathrm{C}$.

\section{N-DOPED Ge-Se-Sb High Temperature StabiLity ANALYSIS}

N-doped Ge-Se-Sb based Crossbar-like OTS devices analyzed in this work are fabricated by sandwiching the OTS layer between a bottom electrode made by a tungsten plug of $350 \mathrm{~nm}$ of diameter and a carbon top-electrode [8]. OTS material and top electrode are deposited by magnetron sputtering without vacuum break in order to avoid surface oxidation. For N-doping we used a mixture of $\mathrm{Ar}$ and $\mathrm{N}_{2}$ as

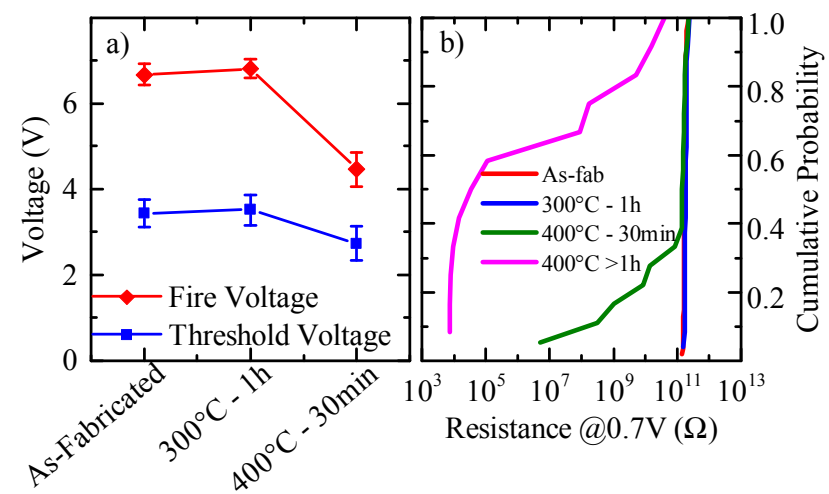

Fig. 1 : Evolution of a) fire $\left(\mathrm{V}_{\text {fire }}\right)$ and threshold $\left(\mathrm{V}_{\text {th }}\right)$ voltage and $\left.\mathrm{b}\right)$ OFF-state resistance after different annealing temperatures. A population of 50 devices is considered for each test reported in this study. 


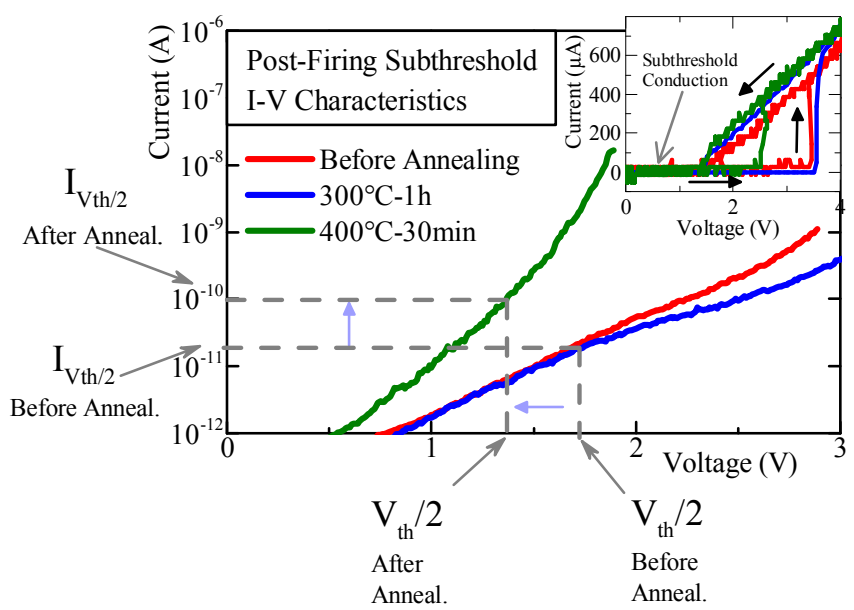

Fig. 2: Evolution of the post-firing I-V characteristics after different annealing steps. A leakage current $\left(\mathrm{I}_{\mathrm{vth} / 2}\right)$ slightly higher than $10 \mathrm{pA}$ is measured before and after the annealing at $300^{\circ} \mathrm{C}$. After the annealing of $30 \mathrm{~min}$ at $400^{\circ} \mathrm{C}, \mathrm{I}_{\mathrm{Vth} / 2}$ slightly increases up to $\sim 0.1 \mathrm{nA}$. The inset shows the full OTS characteristics (pulse mode) for each annealing step.

reactive sputtering gas. The whole fabrication process temperature never crosses $250^{\circ} \mathrm{C}$, allowing the study of the impact of higher temperatures on device performance. In parallel, corresponding OTS thin layers for physico-chemical characterization were deposited at room temperature.

\section{A. Device Electrical Parameters}

As previously reported in the literature, OTS devices need to be initialized by a "fire voltage" $\left(\mathrm{V}_{\text {fire }}\right)$ before achieving regime operating parameters [9][10] (i.e. stable $\mathrm{V}_{\text {th }}$ ). Since fabrication thermal budget could affect this parameter, we studied its evolution, in addition to that of $\mathrm{V}_{\text {th }}$. All the devices electrical parameters are acquired by pulse mode characterization, if not differently specified.

The evolution of $\mathrm{V}_{\text {fire }}$ and $\mathrm{V}_{\text {th }}$ after different annealing steps is reported in Fig. 1(a). After $1 \mathrm{~h}$ at $300^{\circ} \mathrm{C}$, we observe only a slight increase of $\mathrm{V}_{\text {fire }}$ (from $6.7 \mathrm{~V}$ to $6.8 \mathrm{~V}$ ) and $\mathrm{V}_{\text {th }}($ from $3.4 \mathrm{~V}$ to $3.5 \mathrm{~V}$ ). On the contrary, after 30 minutes at $400^{\circ} \mathrm{C}$, both $\mathrm{V}_{\text {fire }}$ and $\mathrm{V}_{\text {th }}$ decrease, respectively down to $4.5 \mathrm{~V}$ and $2.7 \mathrm{~V}$, with a reduction of the $\mathrm{V}_{\text {fire }} / \mathrm{V}_{\text {th }}$ ratio from $\sim 2$ down to $\sim 1.6$. The virgin OFF-state resistance measured at $0.7 \mathrm{~V}$ (Fig. 1(b)) is not affected by the annealing at $300^{\circ} \mathrm{C}$, whereas the annealing of 30

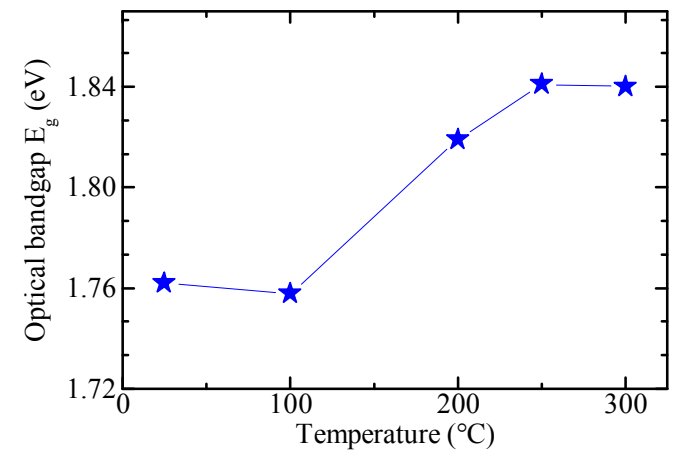

Fig. 3: Evolution of the optical bandgap $\left(\mathrm{E}_{\mathrm{g}}\right)$ after different annealing steps at increasing temperatures. The calculation was performed by fitting the spectroscopic ellipsometry technique results using the Cody-Lorentz model. For each annealing, the heating and cooling rate is $30^{\circ} \mathrm{C} / \mathrm{min}$ and the maximum temperature is maintained during $1 \mathrm{~min}$.

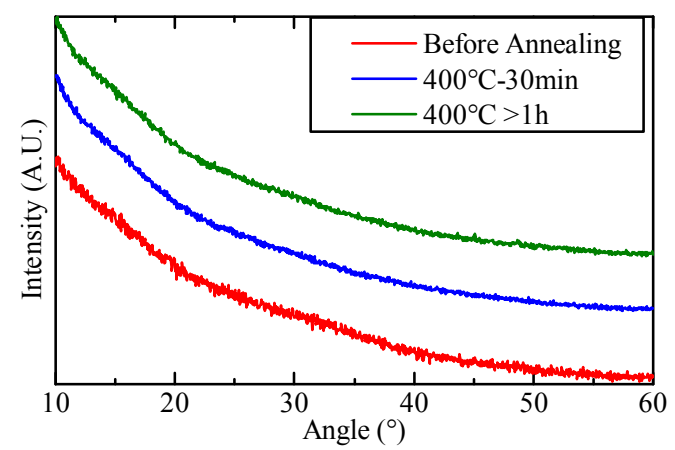

Fig. 4: XRD measurements performed on full-sheet $\mathrm{N}$-doped $\mathrm{Ge}-\mathrm{Se}-\mathrm{Sb}$ material. No crystalline peak is detected even after an annealing of more than $1 \mathrm{~h}$ at $400^{\circ} \mathrm{C}$. Device failure observed in Fig. 1(b) appears not correlated with a bulk crystallization of the OTS material.

$\min$ at $400^{\circ} \mathrm{C}$ induces a reduction of the resistance in $<40 \%$ of the devices population. A longer annealing at $400^{\circ} \mathrm{C}(>1 \mathrm{~h})$ leads to the loss of the high OFF resistance in almost all the devices.

In order to quantify the leakage current in a "V/2" scheme, we considered the leakage current at $\mathrm{V}_{\mathrm{th}} / 2\left(\mathrm{I}_{\mathrm{Vth} / 2}\right)$. In Fig. 2, we plot the post-firing subthreshold quasistatic current-vs-voltage (I-V characteristics) mean curves, after the different annealing steps. A leakage current slightly higher than $10 \mathrm{pA}$ is measured before and after the annealing at $300^{\circ} \mathrm{C}$. In this case, a change in the conductivity is observed only at voltages higher than $1.7 \mathrm{~V}$ (i.e. equal to $\mathrm{V}_{\mathrm{th}} / 2$ before the annealing at $400^{\circ} \mathrm{C}$ ). After the annealing of 30 minutes at $400^{\circ} \mathrm{C}, \mathrm{I}_{\mathrm{Vth} / 2}$ increases from about $0.01 \mathrm{nA}$ to $0.1 \mathrm{nA}$, gaining less than one order of magnitude wrt the value before the annealing steps. Despite this increase, the found leakage current is among the lowest reported so far for a backend selector device [11].

\section{B. MATERial CHARACTERIZATION}

In order to understand the origin of the electrical parameters evolution during the different annealing steps, we performed the physico-chemical analysis on thin N-doped $\mathrm{Ge}-\mathrm{Se}-\mathrm{Sb}$ full sheet amorphous layers. We calculated the optical bandgap $\left(\mathrm{E}_{\mathrm{g}}\right)$ evolution after each annealing step, by means of spectroscopic ellipsometry technique performed in the $300-1800 \mathrm{~nm}$ spectral region and using a Cody-Lorentz (CL) oscillator (Fig. 3). The $\mathrm{E}_{\mathrm{g}}$ values are extracted from the fitting parameters of the

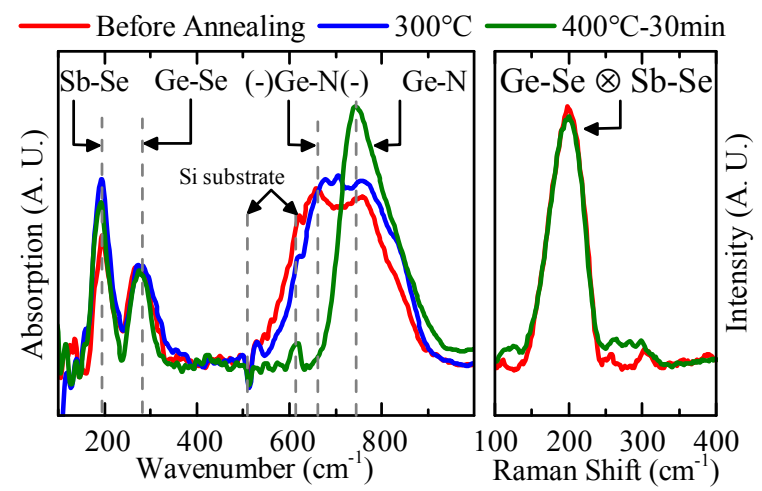

Fig. 5: IR and Raman spectroscopy spectra performed after different annealing steps on Ge-Se-Sb OTS materials. IR spectrum at $300^{\circ} \mathrm{C}$ highlights the drift of the layer, while the one at $400^{\circ} \mathrm{C}$ reveals a rearrangement of the structure (i.e. Ge-N bonds). 


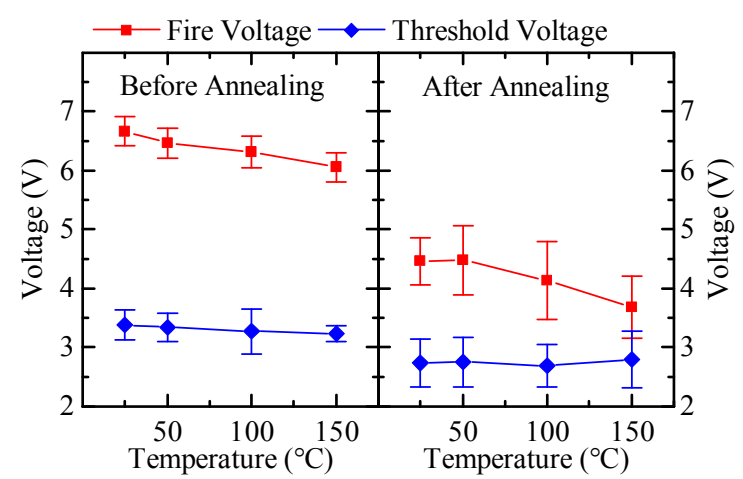

Fig. 6: $\mathrm{V}_{\text {fire }}$ and $\mathrm{V}_{\text {th }}$ values for the OTS devices before and after a $30 \mathrm{~min}$ annealing at $400^{\circ} \mathrm{C}$, measured under a stress temperature up to $150^{\circ} \mathrm{C}$.

ellipsometry measurements using the CL model. $\mathrm{E}_{\mathrm{g}}$ increases up to $300^{\circ} \mathrm{C}$ and this can be related to a structural relaxation typical of an amorphous chalcogenide material (drift phenomenon) [12]. This relaxation is linked to the defects density reduction, boosted by the increase of the temperature, and it leads to the increase of $V_{\text {fire }}$ after the annealing at $300^{\circ} \mathrm{C}$ observed in Fig. 1. Higher temperature $\mathrm{E}_{\mathrm{g}}$ measurements were hindered by the gradual degradation of the encapsulation layer.

No crystalline peaks are detected in the X-Ray Diffraction measurements reported in Fig. 4, even after an annealing at $400^{\circ} \mathrm{C}$ during more than 1 hour. It suggests that the origin of the device failure observed in Fig. 1(b) should not be found in the bulk crystallization of the OTS material, but likely in its interaction with the surrounding co-integrated materials (e.g. electrodes, dielectrics, etc.).

The evolution of the material structure can be further analyzed in the Infrared (IR) and Raman spectroscopy spectra reported in Fig. 5. The slight difference between the spectra of the material as-deposited (before annealing) and after the annealing at $300^{\circ} \mathrm{C}$ could be representative of the already observed drift phenomenon. It affects all the highlighted bonds, and in particular the ones that correspond to Ge-N (present in a $\mathrm{GeN}_{\mathrm{x}}$ phase) and -Ge-N- (bond present as a structural motif linked to the $\mathrm{Ge}-\mathrm{Se}-\mathrm{Sb}$ phase). Indeed, $\mathrm{N}$ atoms are randomly distributed within the $\mathrm{Ge}-\mathrm{Se}-\mathrm{Sb}$ amorphous phase. After the annealing at $400^{\circ} \mathrm{C}$ we observe a reconfiguration of the Ge-N patterns (only the single Ge-N peak is detected), which is likely the consequence of the creation of a stable $\mathrm{GeN}_{\mathrm{x}}$ phase in the layer. The Raman spectroscopy confirms that no homopolar

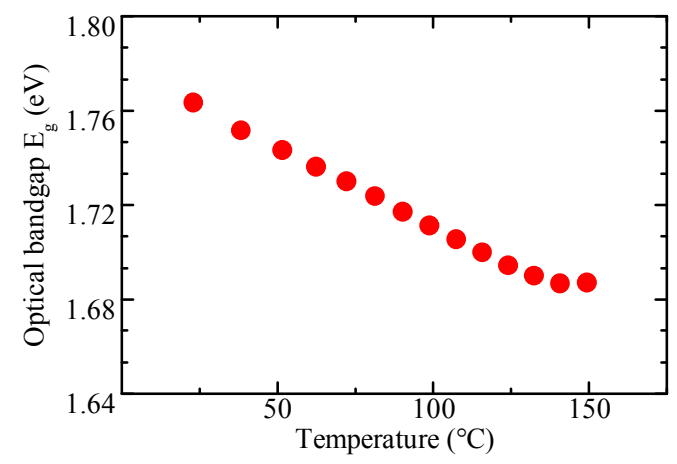

Fig. 7: Evolution of the optical bandgap $\left(\mathrm{E}_{\mathrm{g}}\right)$ of as-deposited full sheet material during in-situ temperature stress. $E_{\mathrm{g}}$ show a linear decrease with the temperature (as observed in Fig. 6 for $\mathrm{V}_{\text {fire }}$ ).

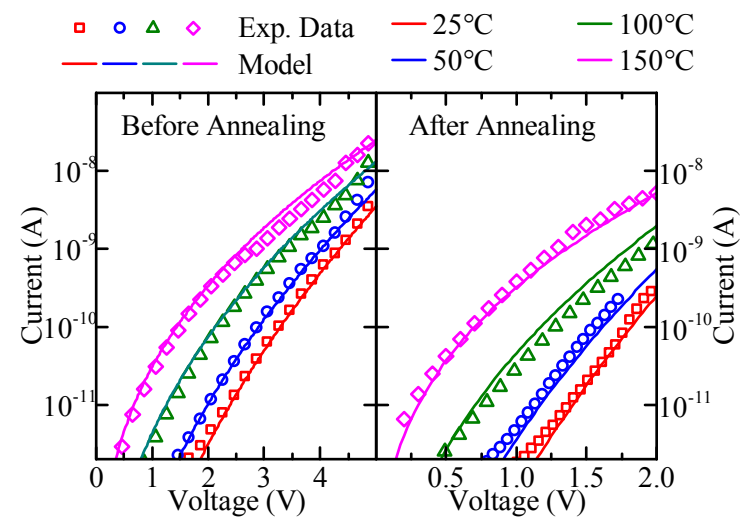

Fig. 8: Subthreshold conduction measured at different stress temperatures, before and after the $30 \mathrm{~min}$ annealing at $400^{\circ} \mathrm{C}$ on as-fabricated devices. PooleFrenkel model is used to model the conduction. The fitted parameters are reported in Table 1.

bonds appear after the annealing at $400^{\circ} \mathrm{C}$ (which could have led to material partial or total crystallization).

The advantageous reduction of the ratio $\mathrm{V}_{\text {fire }} / \mathrm{V}_{\text {th }}$ observed in Fig. 1a after the annealing at $400^{\circ} \mathrm{C}$, could be related to a better structural rearrangement of the material. Therefore, we think that the firing operation leads the material to a rearrangement (thanks to the Joule heating), similar to the one observed after the applied annealing at $400^{\circ} \mathrm{C}$. It means to us that the structure engineering (e.g. deposition parameters tuning) is as much important as the composition engineering for an OTS device with stable performance since first switching operation ("fire-free device").

\section{ELECTRICAL PARAMETERS EVOLUTION UNDER TEMPERATURE STRESS}

The change of $\mathrm{V}_{\text {fire }}$ shows a similar decreasing trend with increasing stress temperature; moving from room temperature up to a stress temperature of $150^{\circ} \mathrm{C}$, before and after the 30 minutes annealing step at $400^{\circ} \mathrm{C}$ (Fig. 6). The measurement of $\mathrm{E}_{\mathrm{g}}$ (in-situ measurement wrt ex-situ measurement of Fig. 3) during an equivalent heating of a full-sheet OTS layer up to $150^{\circ} \mathrm{C}$ exhibits the same linear decrease (Fig. 7). It confirms the strong link between $\mathrm{V}_{\text {fire }}$ and the electronic properties of the material. After the firing, the threshold voltage is almost stable up to $150^{\circ} \mathrm{C}$ both before and after $400^{\circ} \mathrm{C}$ annealing.

OTS materials subthreshold electrical conduction can be described by Poole-Frenkel (PF) model [9][13]. The current density $\mathrm{J}$ follows equation (1):

$$
J=\sigma_{0} \times E \times \exp \left(\frac{-E_{a}}{K_{b} T}\right) \times \exp (\beta \times \sqrt{E})
$$

Where: $\sigma_{0}$ is the zero field conductivity; $\mathrm{E}$ is the electric field; $\mathrm{E}_{\mathrm{a}}$ is the energy barrier that can be considered as the energy

Table 1: Parameters of the fitting by Poole-Frenkel model of the I-V characteristics of Fig. 8.

\begin{tabular}{|c|c|c|c|c|}
\cline { 2 - 5 } \multicolumn{1}{c|}{} & $\sigma_{0}[\mathrm{~S} / \mathrm{m}]$ & $\mathrm{E}_{\mathrm{a}}[\mathrm{eV}]$ & $\beta[\sqrt{(\mathrm{m} / \mathrm{V})}]$ & $\mathrm{E}_{\mathrm{g}}[\mathrm{eV}]$ \\
\hline $\begin{array}{c}\text { As- } \\
\text { fabricated }\end{array}$ & $\mathrm{e}^{6.803}$ & 0.824 & $-6.195 \times 10^{-4}+\frac{4.632 \times 10^{-5}}{K_{b} T}$ & 1.762 \\
\hline $\begin{array}{c}\text { After } \\
400^{\circ} \mathrm{C} \\
30 \mathrm{~min}\end{array}$ & $\mathrm{e}^{17.751}$ & 1.195 & $-1.993 \times 10^{-3}+\frac{1.017 \times 10^{-4}}{K_{b} T}$ & 1.659 \\
\hline
\end{tabular}




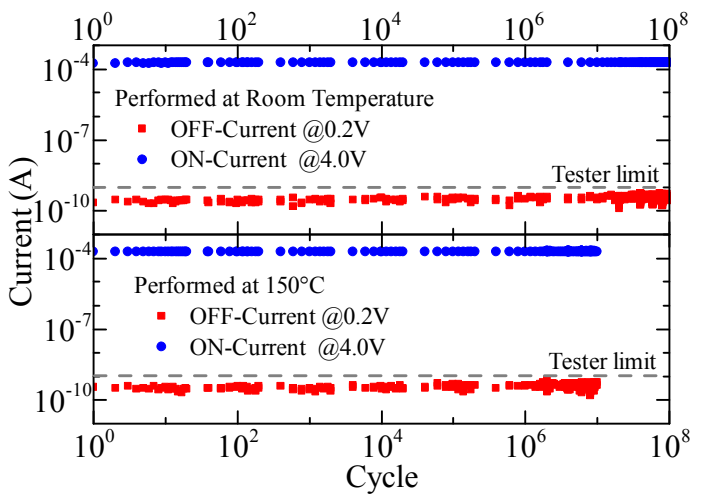

Fig. 9: Endurance test performed after a $30 \mathrm{~min}$ annealing at $400^{\circ} \mathrm{C}$, at room temperature and under a temperature stress at $150^{\circ} \mathrm{C}$. OTS devices are capable of $10^{8}$ cycles even after annealing (pulse width $=500 \mathrm{~ns}$ ).

difference between the conduction band and the localized states; $\mathrm{K}_{\mathrm{b}}$ is the Boltzmann's constant; $\mathrm{T}$ is the absolute temperature; $\beta$ is the Poole-Frenkel parameter [14]. Fig. 8 reports the quasistatic $\mathrm{I}-\mathrm{V}$ characteristics performed at different temperatures (from $25^{\circ} \mathrm{C}$ up to $150^{\circ} \mathrm{C}$ ) on as-fabricated devices before and after the $30 \mathrm{~min} 400^{\circ} \mathrm{C}$ annealing, fitted with a PF model (solid lines). The parameters extracted from the fitting are reported in Table 1. The comparison of the calculated activation energy of $\sim 0.82 \mathrm{eV}$ (before annealing) and the optical bandgap of $\sim 1.76 \mathrm{eV}$ (Fig. 3), suggests that the energy of the localized states is located near the middle of the forbidden gap, as previously reported for this class of materials [15]. After the annealing, the activation energy of the electrical conduction increases from $\sim 0.82 \mathrm{eV}$ up to $\sim 1.20 \mathrm{eV}$. We believe that it can be due to the vanishing of the localized states present in the middle of the gap, which could be generated by a high level of disorder suggested by -Ge-N- patterns observed in IR spectrum (Fig. 5), as well disappeared after the annealing.

\section{N-DOPED Ge-Se-SB OTS Post-ANNEALING PERFORMANCE}

We investigated the endurance performance of $\mathrm{N}$-doped Ge-Se-Sb OTS devices after a $30 \mathrm{~min} 400^{\circ} \mathrm{C}$ annealing (Fig. 9) and we acquired the I-V characteristics at the beginning of the test and after $10^{7}$ cycles (Fig. 10). ON-state current was limited at $\sim 0.2 \mathrm{MA} / \mathrm{cm}^{2}$. Even after $10^{8}$ cycles, the device exhibits an ultra-low leakage current $(<0.1 \mathrm{nA} @ 1.5 \mathrm{~V})$, close to the post-firing one. This result is $\sim 2$ orders of magnitude smaller than the previous leakage current reported for an OTS device sustaining such high annealing temperature [16]. The same test was also performed at $150^{\circ} \mathrm{C}$. An endurance of more than $10^{7}$ is ensured, despites an increase of the leakage current is observed during the cycling.

\section{CONCLUSIONS}

In this paper, we investigate the stability and the performance at high temperature of $\mathrm{N}$-doped $\mathrm{Ge}-\mathrm{Se}-\mathrm{Sb}$ based Ovonic

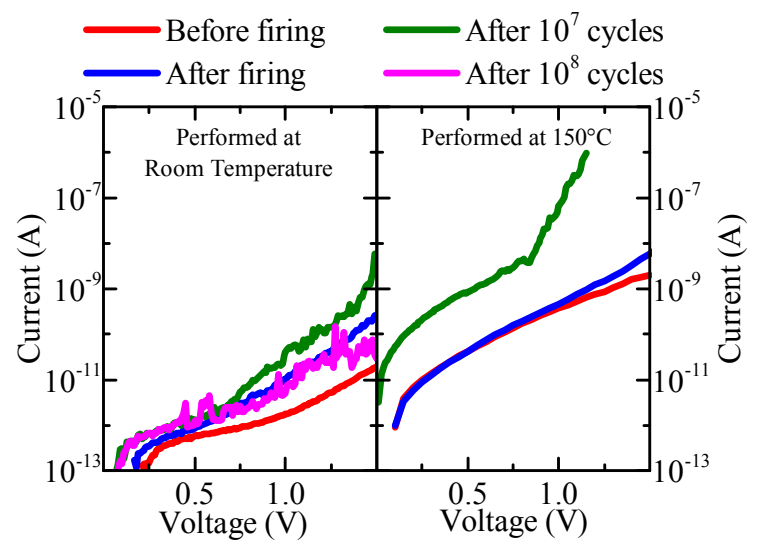

Fig. 10: I-V characteristics performed during the endurance test of Fig. 9. An ultra-low leakage current is ensured even after $10^{7}-10^{8}$ cycles.

Threshold Switching selectors (OTS). Thanks to electrical characterization and material physico-chemical analysis, we show how a BEOL-like thermal budget of 30 minutes at $400^{\circ} \mathrm{C}$ is beneficial for the reduction of the threshold voltage of the device. We demonstrate that this is achieved by a better structural rearrangement of the material, otherwise achieved only through an initialization firing procedure. After such thermal budget, an endurance of more than $10^{8}$ cycles and an ultra-low leakage current of $0.1 \mathrm{nA}$, among the lowest reported so far for a backend selector device, are still ensured. Finally, OTS selector operations are demonstrated up to $150^{\circ} \mathrm{C}$, showing the suitability of Ge-Se-Sb based OTS selector devices for BEOL integrations, even for applications that target high temperature operating environments.

\section{ACKNOWLEDGMENT}

This work has been partially supported by the European 621217 PANACHE Project.

\section{REFERENCES}

[1] X. Peng et al., J. Comput. Electron, vol. 16 (4), pp. 1167-1174, 2017

[2] G. Burr et al., J. Vac. Sci. Technol. B, vol. 32 (4), pp. 040802, 2014

[3] S. R. Ovshinsky, Phys. Rev. Lett., vol. 21 (20), 1968.

[4] http://www.techinsights.com/about-techinsights/overview/blog/ intel-3D-xpoint-memory-die-removed-from-intel-optane-pcm/

[5] A. Velea et al., Scientific Reports, vol. 7 (8103), 2017

[6] J. A. Savage et al., Infrared Phys., vol. 5, pp. 195-204, 1965

[7] A. Verdy et al., 2017 IEEE $9^{\text {th }}$ IMW, pp. 1-4, 2017

[8] A. Verdy et al., 2018 IEEE IRPS, accepted, 2018

[9] N.S. Avasarala et al., 2017 47th ESSDERC, pp. 168-171

[10] S.B. Bhanu Prashanth et al., Solid State Commun., vol. 147 (11-12), pp. 452-456, 2008

[11] S.H. Jo et al., 2014 IEEE IEDM, pp. 6.7.1-6.7.4, 2014

[12] P. Noé et al., Semicond. Sci. Technol., vol. (33), pp. 013002, 2018

[13] M.-J. Lee et al., 2012 IEEE IEDM, pp. 2.6.1-2.6.3, 2012

[14] F.-C. Chiu, Adv. Mater. Sci. Eng., vol. 2014 (578168), 2014

[15] J. Luckas et al., J. Mater. Res., vol. 28 (9), pp. 1139-1147, 2013

[16] Y. Koo et al., IEEE Electron Device Lett., vol. 38 (5), pp. 568-571, 2017 